\title{
Identification of Normal and Abnormal Mammographic Images Using Deep Neural Network
}

\author{
G S Pradeep Ghantasala \\ Chitkara University Institute of Engineering and Technology, \\ Chitkara University, \\ Punjab, India \\ ggs.pradeep@chitkara.edu.in
}

\author{
Nalli Vinaya Kumari \\ Malla Reddy Institute of Technology and Science, \\ Hyderabad, \\ Telangana, India \\ v.vinayakumari@gmail.com
}

\begin{abstract}
This article presents an image retrieval strategy that considers the similarity of a selected image (CBIR-Content-Based Image Retrieval). The analogy is defined by the wavelet technique, combined with Hu moments, for removing features. The classification of mammography's is conducted through the Artificial Neural Networks Auto-organizing System (SME). A Data Base (QUALIM), University of the Federal Republic of São Paulo (UNIFESP), and Medical Image Classification Laboratory are used to test the method. The system suggested is tested. We have employed two widely used pattern recognition approaches-facial recognition digital mammograms for examination. The techniques are based on the new classification schemes Ada Boost and Support Vector Machines (SVM).Several experiments were carried out to evaluate the accuracy of these two algorithms in different circumstances. The results for the Ada Boost classification system are positive, especially for mass lesions classification. In all cases, the algorithm was $76 \%$ exact, and only $\mathbf{9 0 \%}$ accurate for mass. The SVM-based algorithm was not available. To improve the precision of the process, we need to choose enhanced image functionality for digital mammograms than those commonly used. Detection of breast cancer is the most challenging aspect in the field of health monitoring. This document has been used to evaluate breast cancer detection through a dataset of the mammographic image analysis company (MIAS). The suggested method has four key steps: preprocessing, segmentation, retrieval, and classification of images. Initially in mammograms, laplacian filtration was used to describe the edges' area and was thus particularly susceptible to noise. The modified adjustable Fuzzy-C-MEANS (ARKFCM) was used in the following segmentation to find the object within the complicated module. The conventional ARKFCM masses of undefined mass were painful to divide into mammograms. The Euclidean distance in ARKFCM was replaced with the correlation function to solve this problem to increase its segmental efficiency. In the segmented cancer region, the removal of representative subsets was performed by extracting hybrid properties (histogram of guided grade (HOG), uniformity, and energy). Each feature value was defined for the Deep Neural classifier-network (DNN) for detection in normal and pathological areas of mammograms. The findings of the study show that the technique shows an improvement of up to 39\% in precision compared with other methods currently in use in breast cancer classifications. Describes a new way to identify borders between different brightness areas. The goal is to create distinctions between regions that are unclearly distinct and defined by tonal shifts. The limits are set by the coordinates of the start and endpoints. The proposed method can be used as a stage in advanced techniques for hierarchical image analysis that increases the semantically awareness of picture content with each
\end{abstract}

step. This study applies and evaluates mammograms. Interpretation of mammograms is a complex research area discussed by many authors. The treatment of breast cancer is achieved using these approaches.

Keywords- Deep Neural classifier, Mammogram, Pattern Recognition, Euclidean distance

\section{INTRODUCTION}

Wilhelm Conrad Röntgen was able to imagine the internal mechanisms for the human body in 1895, following the exploration of X-rays by a German scholar [1]. Lehmann has stated that machine pictures and computer technologies have been established in recent years [2,13]. Digital information can be processed in computer tomography (CT) and digitally generated magnetic resonance (MR) images [3]. Photos can be seen on computer monitors, including post-processing tools. Digital image processing $[4,15]$ is an issue in medical and biomedical engineering. The challenge in measuring the output of the different methodologies is one of the problems found during data processing. Imaging digital medical photographs can be stored for printing or diagnosis in various media.

The increase in the use of images by radiologists has contributed to the creation of the concept of PAQS [3]. PACS's interconnected image processing and viewing programs enable the distribution of photos over a wireless network [6] as part of the paper. A medical picture study and analysis in the QUALIM laboratory of UNIFESP is being performed to educate people for accurate diagnostics. One of the problems in training processes is that it is difficult to detect and differentiate the diagnostic object with people with similar pathologies. A technique for content-based image retrieval (CBIR) has been studied in the last few years [6]. These approaches are used to identify associated images by Data Mining. Data mining is a technology used to disclose large amounts of information to strategic data. The image recovery system uses content indexing based on image visual content assessment. It is used in a range of fields, including Risk assessment, targeted marketing, quality control, and scientific data analysis. Data mining identifies and derives meaning in the automated processing and review of large data sets. It is used to describe the characteristics of the past and to predict future trends. Many studies on the use of data mining as a diagnostic aid in the medical field are carried out. In medical images, Antoine employed methods of data mining to identify 
tumors [7]. We emphasize the following aspects in preparing reports from the images:

- Parallel's that cannot be rendered with other science images; accuracy and validity of data sets for two or more experiments tend to produce identical test results. If there is any disparity, a separate physician must perform the test whenever possible. If it remains the disparity, the photos for training will be removed from the images;

- The ability to use processing forms automatically;

- Tracking of training challenges for residents;

- The complexity required for the learning stage of the residents is difficult to use previously selected images.

\section{Motivation AND RATIONALE}

In several research centers, software programs are being developed to identify evidence that matches the presence of tumors. Negative mammograms range between 10 and 30 percent for women with breast cancer and mammography [8,11,12]. For specialists, mammography lesions are hard to diagnose because the contrast between mammographic images is low and other quality factors. The most effective method to detect breast cancer is mammography. In mammograms, it is relatively unproblematic to classify lesions as malignant or benign [9,14]. The São Paulo Federal University, Diagnostically Imaging Institute, provides research in radiology in the Diagnostic Imaging Skills Laboratory (QUALIM) for medical residents. You define the visual imaging modes, and the tests are compared to those of professional doctors. Their nature can categorize digital images through identification and pattern exploration. This research focuses on the automated detection of the regularity of data using computer algorithms to ensure data is consistently sorted into different categories [10]. We consider the machinery training approach in which a collection of training data is used to establish decision-making criteria to enhance classification performance. Training can be tracked or not. The objective of supervised learning is to explain interactions and the number of unknown outcomes [11], while data and classes are known. In an analysis, an unattended. This study's purpose is to suggest a methodology for the report by radiological residents of mammographic photographs. This proposal starts with the development of an IT System already described for the purposes. To do this, we have created an application that uses imaging techniques in combination with $\mathrm{Hu}$ moments, including Wavelet Transformation, to identify and classify similar images. Data mining methods were used to identify mammographic images eventually. To identify such datasets in the class, pattern recognition is structured according to similarities.

Classification takes place through the self-organization map of the Artificial Neural Networks [12]. The accuracy of classification can be achieved by field testing in the Receiver Operating Features (ROC). Considering the resemblance of the previously chosen image for testing, the device allows image recovery based on quality. Image Recovery for CBIR ContentBased. Similarity can be defined with the extraction of texture and shape characteristics by using the transition wavelet. A variety of wavelets are evaluated to see the right wavelet for this function as per Silva's work [12]. The diagnosis of experienced doctors is included in the training of radiology residents, and complexity can be taken in the selection of clinical cases. Each resident must be trained in various degrees of difficulty so that the assessment can be identified according to training trends. In comparison, diagnostic photographs will also be used in another instruction for the area designed initially for educating residents in breast radiology.

\section{WORK PROPOSAL}

This research suggests a reinforcement of medical graduates' radiology preparation. The proposal aims to display similar images using CBIR and segmented images containing areas of interest identified by practitioners of experience. The object of the scheme is to give the picture the same characteristics. Details identified by doctors when preparing reports can also be seen in addition to residents. MammoAdviser is the current medical reporting system for selected mammograms. -- Mammogram is reviewed by two qualified physicians, who are just the physicians of the name. A final review is done where there are inconsistencies. Unless that is not necessary, the mammograph is not used for training purposes.

Residents are available after the information has been generated on medical pictures. This teaching system [13] has as its primary purpose:

- A strong interface between person and machine;

- To allow professional outcomes to be contrasting;

- Develop phrases that are sensitive to the individual professional problems; an independent image classification module needs to work when new images are inserted in the database. The modular design enabled MammoAdviser to be extended with new modules. This research and health preparation was developed by doctors to produce the regular report for the implementation of MammoAdviser.

The diagnostic planning checks the doctors' performance in keeping with the daily analysis. The MammoViewer module aims at displaying medical pictures of the same kind and views grouped into areas of concern after the introduction of the regular study by doctors. The doctor responsible for reporting can detect an anomaly in the default Mammoadviser module.

Core fields of concern, namely segmented areas, are managed by doctors. The tenants should navigate such regions. For the submission, MammoSort sortes pictures. The images are processed at this stage by comparing the material (CBIRImage Retrieval) in conjunction with the resemblance of an earlier selected image. The collection of the picture is linked to people's learning. The images that have been obtained by the CBIR for analysis cannot meet the physician's standards. The semi-anticized separation is called this division. Several experiments to classify the extractor function have been conducted to boost the classification performance and image recovery accuracy. To extract the medical image features, the Wavelet Transform is used. BI-RADS (B) with the 
SelfOrganizing Maps (SOM) Artificial Neural Network is responsible for organizing photos [14]. Several automated classification schemes may be predictive such as Knn[11], or involve learning to identify resemblance images[15]. We used the SOM Neural Network based on research by Silva[12] and which has benefits such as unattended study, clustering, and browsing search. Differences are detected using the transformation of the wavelet to extract the characteristics of texture and form. The most effective wavelet is done for this purpose. The residual analysis and the interpretation of pictures of the same resemblance are integrated into the preparation in radiological testing. The degree of difficulty may be used to identify clinical cases. Depending upon the formation development, each resident has to be accompanied by difficulty diplomas in determining the evaluation.

Diagnostic photos may be used in other education schemes to provide educators for breast residents planned initially.

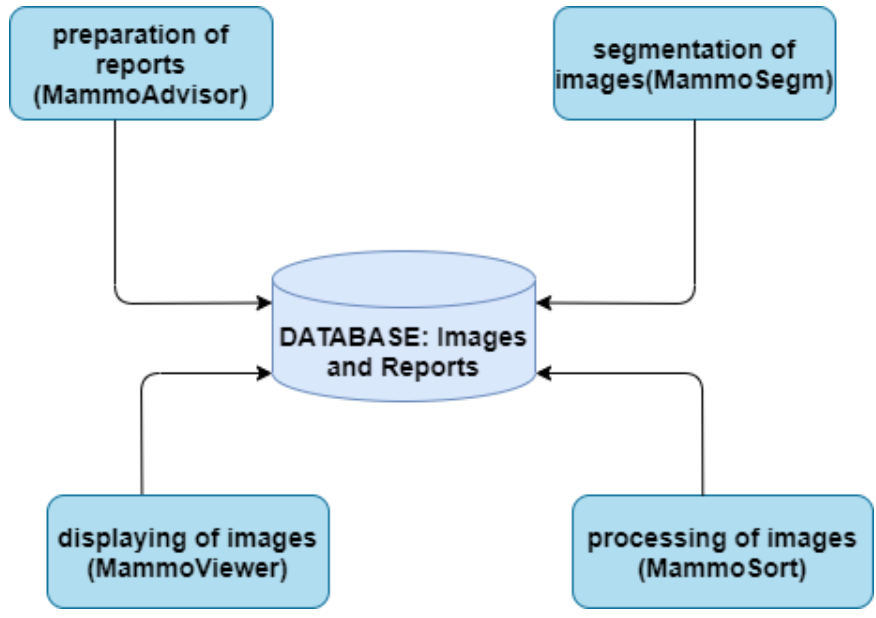

Fig. 1. Proposed Architecture

\section{A. Overview of the architecture}

The suggested architecture involves the MammoSegm Image Segmentation (ROI), the MammoSegm Image Similarity Classification Modul (MammoSort), and the MamMoDefinition Module that displays images, identified as MammoViewer and MammoAdviser that produces a diagnostic record in the database (Figure 1). The first photos of the papers go into a test and classification to scan for resemblance. The resident doctors will carry out their preparation with the study prototype and the images stored. After recording, the citizen may ask the program to show pictures with the same complexity and consistency (It is possible to view the image of a given reporting area in the database). Then the report is in the database. To determine by resemblance, the original photos of the papers are analyzed and categorized. With the study prototype and the produced pictures, the resident doctors shall conduct their preparation. After the query is performed, the citizen can request that an image in the database be viewed to the same sophistication and consistency (if the image has a particular object field in the database the image is displayed). Both records are held separately or jointly in the citizens' statistical appraisal information program. The solution is split into two parts. Netbeans are used as a networking tool for the end-user using the Java programming language ® (SUN 2006). The Java ecosystem benefits from simplicity, portability, code reuse, and fast programming. Jaï (Java Advanced Image) (SUN 2006) API (API) enables the classification method, using the MATLAB (B built-in framework, to be performed using Artificial Neural Network, which has been used in conjunction with this programming language.

\section{B. Physicians first examine the captured images.}

The decisions were made using Munerati's MammoAdviser module (MUNERATI 2011). The findings are applied to the database. The report is considered the reference report, which is used to compare the residents' diagnoses.

Each picture provides a regular study, and if doctors disagree, a separate examination is carried out. Nonetheless, if uncertainty remains, the teaching base will be withdrawn.

\section{Picture segmentation}

Physicians complete the report, and the MammoAdviser updates the database. To help train resident doctors using the MammoSegm module, only areas of concern for each of the images can be chosen and produced. The design of the MammoSegm module includes three tables: IMAGEM SEGMENTADA, which stores a segmented image's code and location; IMAM OBSERVACAO, which stores system and related image information and IMAGEM TIPO table, where a piece of anomaly information is stored. The doctor will define a variety of areas of concern for each photograph. The module initially allows nodes and microcalcifications to be established.

\section{Extracting, classifying, and visualizing features:}

The Mammosort module provides an interface for selecting the parameters for the extraction and classification of functions. This module includes a MammoViewer gui that can locate pictures from the database depending on the needs of the residents or also of doctors who conduct a sectioning and testing process for similarities.

\section{PROPOSED METHOD}

The technique used in the research is described in depth below. It covers all preparation stages, feature extraction phase, and classification of medical imaging and updating the base for resident physicians to conduct research. Figure 2 provides an outline of the process operation built.

Each step of the method, the initial phase of pre-processing, is detailed in the following paragraphs.

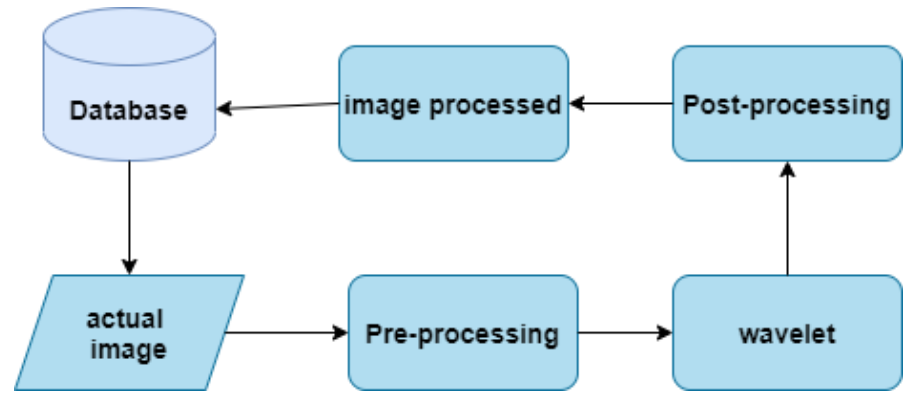

Fig. 2. General overview of the developed technique 


\section{A. Pre-purification}

This period aims at standardizing medical images through gray standardization - the wavelet changes at the end of this step. The entire series of images have been sent to the extractor attributes by transforming the wavelet and the Hu-moments, each image type, mixing $\mathrm{Hu}$ measurement with the discarded wavelet coefficients. This technique was used successfully in Silva[3] for medical photos. For each script, a preprocessing is carried out to improve the pathological field.

\section{B. Stage of Wavelets}

The purpose of the present study is to identify images for medical students. We use an extractor that converts the wavelet and uses a grading map. AMR derives functions through the decomposition of the image in four coefficient sets. Figure 3 shows the criterion for the number of decays used. Tools used for measuring the SOM 's performance include an average error in quantitative quantities, average $\mathrm{MQE}$ error (average termination error), and topographical error and hits[6,16] for each degradation lever.

Initially, the hair wavelet was used to describe failure. After the degree of decomposition is specified, experiments with other wavelet mothers must be conducted. Following the concept of mother wave, the coefficients and characteristics of the training images are collected.

\section{MQE, TE, IDB, and hit-rate chart}

In the MQE, TE, IDB, and hit-rate chart Dimensions are used in the area of $4 \times 4,6$ X6, 8 X 8, 10X10, 12 X 12. SOM SOM Size measurements are used to define features. In this case, a program is split into groups that use the $\mathrm{K}$-media method to calculate the group weight vectors and the IDB. This is achieved with Vesanto and Alhoniemi[18]. The DaviesBoudin Scale (IDB) offers a consistency measure for the clustering. The image properties of the best behavior and the best wave family.

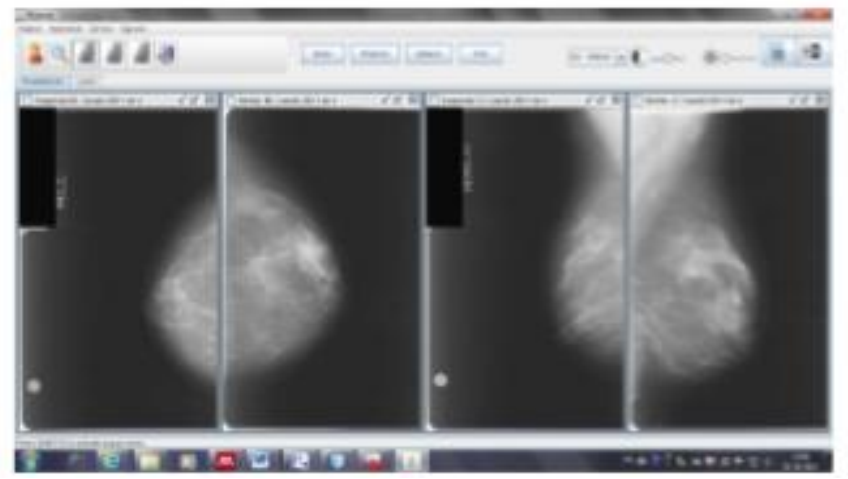

Fig. 3. - Example of system utilization

\section{Post-processing:}

The data generated in previous cases was modified at this point in the database, which would involve updating the established database tables.

\section{CONCLUSION}

The first application of the device recovery based on content-driven radiological photographic instruction is discussed in this article. The program will allow for comparisons of photographs with the same features as a radiologist's work. The architecture is also flexible to enable new classifiers to be introduced to simplify database query procedures.

\section{REFERENCES}

[1] O. Glasser, "Wilhelm Conrad Röntgen and Röntgen Ray 's Early Growth," vol. American Medical Research Review Number 4:187, p. 5661934.

[2] T. Keysers, M. Lehmann, M. Kohnen, H. Schubert. Wine. "IRMA coding for advanced medical imaging," 2003, pp. 440-451.

[3] Re. 59, Dec. 3, 187-88, 2003. M. Siege, E. L.; Kolondner, MJAFI, MJAFI, "Filmless Radiology-A possibility view." 2003. 2003.

[4] C. R. Gonzales, U. R. E. Woods, Manufacture of Digital Image, Terceira. São Paulo, Pearson Prentice Hall, 2010.

[5] M. Santos and S. And S. Two. S. Furuie, Video Clinical Application Computer and Algorithms, 2005, pp. 267-275.

[6] H. D. Bandon, A. N. Michoux, Müller. Geissbuhler, "European Medical IT Magazine," vol. "Review of existing and forward-looking image recovery technology for material." 73 , No. 1, February 2004, pages 1 to 23.

[7] M. R.M. Zaiane, Anthony, A. SIGKDD, Sixth ACM International Knowledge Discovery and Data Mining Conference, 2001, pp. 94-101. Coman, "Introduction of Health Photo Date Mining Techniques."

[8] M. "Computer-aided master injury diagnosis for medical photographs," L. Giger, Code of science and engineering, vol. 1; 5; 39-45, 2000. 5. 2000. 2000

[9] L. H. P. Chan, H. B. Chan, N. Petrick, M. H. P. Chan, Hadyisky. IEEE Medical Imagine transacts Helvie, "Hybrid ART2LDA Classification and Benign Masses," vol. 1. 18, 12, 1999. 1999. 1999. 1999.

[10] C. M. Bishop, Pattern Recognition and computer learning. 2003., 2007. New York.

[11] G S Pradeep Ghantasala et al., International Journal of Research in Engineering, IT and Social Sciences, ISSN 2250-0588, Impact Factor: 6.452, Volume 06 Issue 09, September 2016, Page 50-54

[12] Kumari, Nalli Vinaya, and GS Pradeep Ghantasala. "Support Vector Machine Based Supervised Machine Learning Algorithm for Finding ROC and LDA Region." Journal of Operating Systems Development \& Trends 7, no. 1 (2020): 26-33.

[13] Ghantasala, GS Pradeep, Suresh Kallam, Nalli Vinaya Kumari, and Rizwan Patan. "Texture Recognization and Image Smoothing for Microcalcification and Mass Detection in Abnormal Region." In 2020 International Conference on Computer Science, Engineering and Applications (ICCSEA), pp. 1-6. IEEE, 2020.

[14] Mammographic CADe and CADx for Identifying Microcalcification Using Support Vector Machine. Journal of Communication Engineering \& Systems. 2020; 10(2): 9-16p.

[15] "A survey on Microcalcification identification and classification using CAD System", International Journal of Emerging Technologies and Innovative Research (www.jetir.org), ISSN:2349-5162, Vol.2, Issue 5, page no.186-190, MAY-2015, Available :http://www.jetir.org/papers/JETIR1805783.pdf

[16] Ghantasala, GS Pradeep, Suresh Kallam, Nalli Vinaya Kumari, and Rizwan Patan. "Texture Recognization and Image Smoothing for Microcalcification and Mass Detection in Abnormal Region." In 2020 International Conference on Computer Science, Engineering and Applications (ICCSEA), pp. 1-6. IEEE, 2020. 